\title{
Serum Cytokines Profile in Treated Celiac Disease Compared with Non-celiac Gluten Sensitivity and Control: a Marker for Differentiation
}

\author{
Fatemeh Heydari ${ }^{1,2}$, Mohammad Rostami-Nejad ${ }^{2}$, Ali Moheb-Alian ${ }^{3}$, Mostafa Haji Mollahoseini ${ }^{1}$, Kamran Rostami ${ }^{4}$, \\ Mohamad Amin Pourhoseingholi ${ }^{2}$, Elham Aghamohammadi ${ }^{2}$, Mohammad Reza Zali ${ }^{1}$
}

1) School of Medicine, Shahid

Beheshti University of Medical

Sciences, Tehran, Iran

2) Gastroenterology and

Liver Diseases Research

Center, Research Institute for

Gastroenterology and Liver

Diseases, Shahid Beheshti

University of Medical Sciences,

Tehran, Iran

3) Basic and Molecular

Epidemiology of

Gastrointestinal Disorders

Research Center, Research

Institute for Gastroenterology

and Liver Diseases, Shahid

Beheshti University of Medical

Sciences, Tehran, Iran

4) Department of

Gastroenterology, MidCentral

District Health Board,

Palmerston North Hospital,

Palmerston North,

New Zealand

\section{ABSTRACT}

Background \& Aims: There is increasing evidence regarding elevated serum levels of inflammatory cytokines in patients with celiac disease (CD), but little is known about their levels in patients with non-celiac gluten sensitivity (NCGS). The aim of this study was to evaluate the serum levels of inflammatory cytokines in Iranian patients with CD and NCGS and to compare them with those of healthy individuals.

Methods: A total of 110 treated CD, 15 with NCGS, and 46 healthy subjects were enrolled during 2016. Serum levels of IL-1, IL-6, IL-8, IL-15 and IFN- $\gamma$ were measured using ELISA, and compared between groups. The correlation of the severity of mucosal damage and clinical symptoms with serum levels of cytokines was also assessed.

Results: The mean serum levels of IFN- $\gamma(\mathrm{p}=0.04)$ and IL-6 ( $=0.007)$ were significantly different between the patients in the CD and control groups, and IL- 8 was significantly higher in the CD group compared with patients in the NCGS group ( $\mathrm{p}=0.04)$. Statistically significant correlations were observed between the serum levels of IFN- $\gamma$ and abortion $(p=0.01)$, IL-1 and weight loss $(p=0.043)$ and infertility $(p=0.0001)$ in CD patients, and between IFN- $\gamma$ and abortion $(\mathrm{p}=0.01)$ and infertility $(\mathrm{p}=0.01)$ in the NCGS patients. Moreover, no significant relationship was observed between the severity of mucosal damage and the serum level of the studied cytokines.

Conclusions: Inflammatory cytokines are implicated in the pathogenesis of CD, and their serum levels might help to identify a diagnostic marker to differentiate CD from NCGS. However, further studies with a larger sample size are recommended.

Key words: Celiac Disease - Inflammatory Cytokines - Non-celiac Gluten Sensitivity - Clinical Manifestations.

Abbreviations: CD: Celiac disease; EMA: Endomysial antibodies; IFN- $\gamma$ : Interferon gamma; GFD: Gluten free diet; GI: gastrointestinal; NCGS: Non-celiac gluten sensitivity; RT-PCR: Reverse transcription polymerase chain reaction; tTG: Tissue-transglutaminase.

\section{INTRODUCTION}

As a multicomponent and immune-mediated intestinal disorder, celiac disease (CD) has a wide spectrum of clinical manifestations. It has been well understood that the ingestion of gluten, mainly found in wheat, rye, and barley, is the main external trigger of $\mathrm{CD}$ in predisposed individuals [1]. To date, the diagnosis of CD [2] is based on serological tests such as anti-tissue transglutaminase (tTG) antibodies and anti- endomysial antibodies (EMA), confirmed by the endoscopy and pathological assessments of small intestine biopsy [3]. In CD, both T helper cell type 1 (Th1) and T helper cell type 2 (Th2) have been reported to be elevated [4]. It has been well established that $\mathrm{CD}$ results from an unwanted T-cellmediated autoimmune reaction. In this reaction, when the modified gliadin-derived peptides are presented through human leukocyte antigen (HLA) molecules DQ2 and DQ8 to CD4+ T helper cells, resulting from the enzymatic activity of tissue tTG, T cells and B lymphocytes get activated, producing anti-gluten and anti-tTG antibodies in lamina propria, which inevitably leads to the activation of autoreactive Th cells [5]. The activation of these autoreactive Th cells leads to the release of proinflammatory cytokines, which in turn activate intraepithelial lymphocytes, thus triggering histological alterations of the small intestinal mucosa. Cytokines involved 
in the pathogenesis of CD [6] affect T cells and other immune-related factors involved in both the suppression and enhancement of the immune-mediated responses [7]. Previous studies have also demonstrated the role of T cell activation in the small intestinal mucosa, promoting interferon-gamma (IFN- $\gamma$ ) producing Th1 cells in the active form of CD [8]. Furthermore, interleukin (IL)-15 induces the activity of innate cells, and the intraepithelial infiltration by these innate cells leads to the secretion of high amounts of a great variety of cytokines, including IFN- $\gamma$ [9]. As an inseparable part of inflammation, the role of IL- $1 \beta$ has also been proposed in mediating mucosal damage [10]. Increased levels of this cytokine stimulate the excessive expression of matrix metalloproteinases (MMPs; mainly MMP-1 and MMP-3), resulting in the degradation of extracellular matrix components, thus contributing to architectural abnormalities [11].

Similar to CD, non-celiac gluten sensitivity (NCGS) as a condition, mainly associated with the ingestion of foods containing rye, barley, and wheat, can manifest various subjective symptoms, including abdominal pain, numbness in extremities, headache, and fatigue, as well as more severe neurological symptoms such as cerebellar ataxia and schizophrenia [12]. Despite designated Salerno criteria for the confirmation of NCGS [13], the exact definition for NCGS remains a controversial issue, and many symptoms in patients without $\mathrm{CD}$ and wheat allergy in response to gluten-free diet (GFD) have been considered to be associated with NCGS. At present, there are no specific diagnostic markers for NCGS. The exclusion of CD by a negative CD serological marker and normal histology, as well as normal specific IgE for the exclusion of wheat allergy, is possible but may not be indicative of NCGS $[14,15]$. Of the above-mentioned factors, HLA genes have been proposed to provide the strongest genetic risk for CD, but not for NCGS, because the majority of CD patients have been shown to carry genes encoding HLA-DQ2 and/or HLA-DQ8, but a positive or negative HLA may not confirm or rule out NCGS $[16,17]$

After the ingestion of gluten, both innate and adaptive immunity are activated in CD. In NCGS, only the innate immunity is proposed to be activated [18]. Therefore, proinflammatory cytokines/chemokines such as IL- $1 \beta$, IL-6, IL-8, IFN- $\gamma$, IL-2, and IL-15 may be engaged in the pathogenesis of NCGS.

Two decades ago, studies were focused on CD cytokine profiles of duodenal and jejunal biopsy samples, at the local level of small intestinal mucosa, using immunohistochemical methods [19]. Few studies have assessed the serum levels of cytokines using enzyme-linked immunosorbent assay (ELISA) at the systematic level [20] and found increased levels of IL2 , IL-18, and IFN- $\gamma$ in patients with active CD. Increased expression levels of IFN- $\gamma$, IL-2, IL-4, and IL-10 mRNA using reverse transcription polymerase chain reaction (RT-PCR) in patients with active CD have also been reported [21]. Moreover, the increased production of tumor necrosis factor (TNF)- $\alpha$ and IL- 8 has also been reported, being stimulated by gliadin peptides [22]. In fact, because both CD and NCGS are associated with inflammatory immune responses [18] and are triggered by the ingestion of gluten, it seems to be necessary to compare inflammatory cytokines between patients with
CD and NCGS for a better understanding of the systemic involvement of inflammatory responses in both diseases and for a possible distinguishing diagnosis tool for NCGS.

Some studies suggested that in patients with active CD, the level of these cytokines might normalize after starting the GFD, but the level of these cytokines in the treated CD as well as NCGS patients has not been investigated [19-22]. In this study, serum levels of IL-6, IL-1, IL-15, IL8, and IFN- $\gamma$ were evaluated in CD and NCGS patients, in comparison with healthy subjects. The correlations between the serum levels of these cytokines and the demographic data of enrolled subjects, as well as the mode of some related clinical presentations were assessed.

\section{METHODS}

\section{Study population and ethical considerations}

This study was conducted in 2016 at the Research Institute for Gastroenterology and Liver Diseases, a referral center for CD in Tehran, Iran. At the department of CD, serum samples of $110 \mathrm{CD}$ patients on GFD for 1 year were collected. The patients had been previously diagnosed following positive EMA and/or TGA, and with histological abnormalities grades 2 and 3 according to the Marsh classification [23]. Their gastrointestinal (GI) and extra-GI symptoms at the moment of diagnosis were collected. Matched subjects without any GI and non-GI disorders were referred for a routine checkup and served as the control group.

Among 200 patients with symptoms suggestive of CD, but negative for both specific biomarkers (anti-tTG and/or anti-EMA antibodies), NCGS was diagnosed in 15 cases. These enrolled patients responded to GFD for 6 weeks and their symptoms returned after gluten challenge. Patients with CD presenting with any symptoms (GI and extra-GI) despite 1 -year GFD and those with a history of any other autoimmune disorders such as inflammatory bowel disease (IBD) and type 1 diabetes were excluded. Written informed consents were obtained from all included subjects, and all procedures were approved by the Medical Ethics Committee of the Research Institute for Gastroenterology and Liver Diseases, Shahid Beheshti University of Medical Sciences, Tehran, Iran.

\section{Collection of data and samples}

Overall, $10 \mathrm{cc}$ peripheral blood was collected from $110 \mathrm{CD}$ patients, 15 patients with NCGS, and 46 healthy subjects from January to December 2016. Sera were isolated and stored at $-80^{\circ} \mathrm{C}$ before testing. Demographic and clinical information of all participants was collected using a standard questionnaire.

\section{Immunology assay}

Serum levels of cytokines including IL-1, IL-6, IL-8, IL-15, and IFN- $\gamma$ were measured using the commercial ELISA kits (all from eBioscience indirect sandwich ELISA kit), according to the manufacturer's instructions.

\section{HLA typing}

DQ2/DQ8 haplotypes were determined using real-time PCR based on the SYBR-Green method as described previously by the authors [7]. 


\section{Statistical analysis}

Differences between the mean values of height and weight, as well as age differences, among the studied groups were evaluated using the Student $t$ test. The distribution of gender proportion among groups and the correlation between the $\mathrm{CD}$ manifestations and serum levels of cytokines were performed using the Fisher exact test and analysis of variance (ANOVA) test, respectively. The Tukey multiple comparison test was performed to analyze the difference between the experiment groups in any ANOVA test. IBM's Statistical Product and Service Solutions, version 20 , were used as a statistical analysis tool in this study. A p value of less than 0.05 was considered statistically significant.

\section{RESULTS}

Mean age of the 110 patients with CD, 38 males (34.5\%), was 33.6 years. Mean age of the 15 NCGS patients, 7 males (46.7\%) was 38.7 years. Mean age of the 46 healthy individuals, 20 males (43.4\%) and 26 females (56.6\%), was 38.8 years.

According to the demographic data, there was no statistically significant difference between the mean age of the studied groups, and the Fisher exact test confirmed no significant difference between the CD and NCGS groups based on gender $(p=0.397)$. Interestingly, statistically significant differences were observed in the heights and weights of the participants in the CD and NCGS groups. The results showed that the mean height for the $\mathrm{CD}$ group was $162.9 \mathrm{~cm}$ in contrast to $170.3 \mathrm{~cm}$ for the NCGS group ( $\mathrm{p}=0.047)$. The mean weight for the CD group was $56.7 \mathrm{~kg}$, significantly less than that of the NCGS group (56.7 vs. 67.3, $\mathrm{p}=0.015$ ). The mean height of males was higher than that of females in all the study groups (male/female for CD, 172.24/156.81; for NCGS, 179/162.75; for control, 165.6/3/155.81). However, these differences were
Table I. The frequency of GI, extra GI symptoms in CD and NCGS patients

\begin{tabular}{lccc}
\hline Clinical Parameters & $\begin{array}{c}\text { CD Group } \\
\text { N (\%) }\end{array}$ & $\begin{array}{c}\text { NCGS Group } \\
\text { N (\%) }\end{array}$ & p value \\
\hline Diarrhea & $43(39.1)$ & $6(40.0)$ & 0.419 \\
Bloating & $90(81.8)$ & $10(66.7)$ & 0.193 \\
Gas passing & $70(63.6)$ & $10(66.7)$ & 0.443 \\
Crumping & $59(53.6)$ & $7(46.7)$ & 0.5 \\
Weight loss & $71(64.5)$ & $7(46.7)$ & 0.183 \\
Nausea and vomiting & $44(40.0)$ & $7(46.7)$ & 0.329 \\
Anemia & $60(54.5)$ & $5(33.3)$ & 0.125 \\
Bone disease & $53(48.2)$ & $10(66.7)$ & 0.177 \\
Neurological symptoms & $41(37.3)$ & $8(56.2)$ & 0.183 \\
Menstrual disorder & $31(28.1)$ & $4(26.7)$ & 0.482 \\
Infertility & $5(4.5)$ & $1(6.7)$ & 0.667 \\
Abortion & $17(15.5)$ & $2(13.3)$ & 0.616 \\
Aphtus & $19(30.2)$ & $4(26.7)$ & 0.53 \\
Skin disease & $19(17.3)$ & $2(13.3)$ & 0.464 \\
\hline
\end{tabular}

CD: Celiac disease; GI: gastrointestinal; NCGS: Non-celiac gluten sensitivity

statistically significant for the CD and NCGS groups ( $\mathrm{p}=0.0001$ for both CD and NCGS groups).

The most prevalent symptoms of the $\mathrm{CD}$ group were bloating and abdominal pain (81.1\%), while in the NCGS group, non-GI symptoms, including bone disease (66.7\%) and neurological problems (56.2\%) (Table I). Moreover, $97 \%$ of the CD group patients were carrying HLA-DQ2 and/or HLA-DQ8 heterodimers, whereas only $26.6 \%$ of the NCGS group patients and $40 \%$ of healthy subjects were carrying DQ2 and/or DQ8 heterodimers, respectively.

Serum levels of cytokines were evaluated in all the three study groups: CD, NCGS, and control. ELISA test results
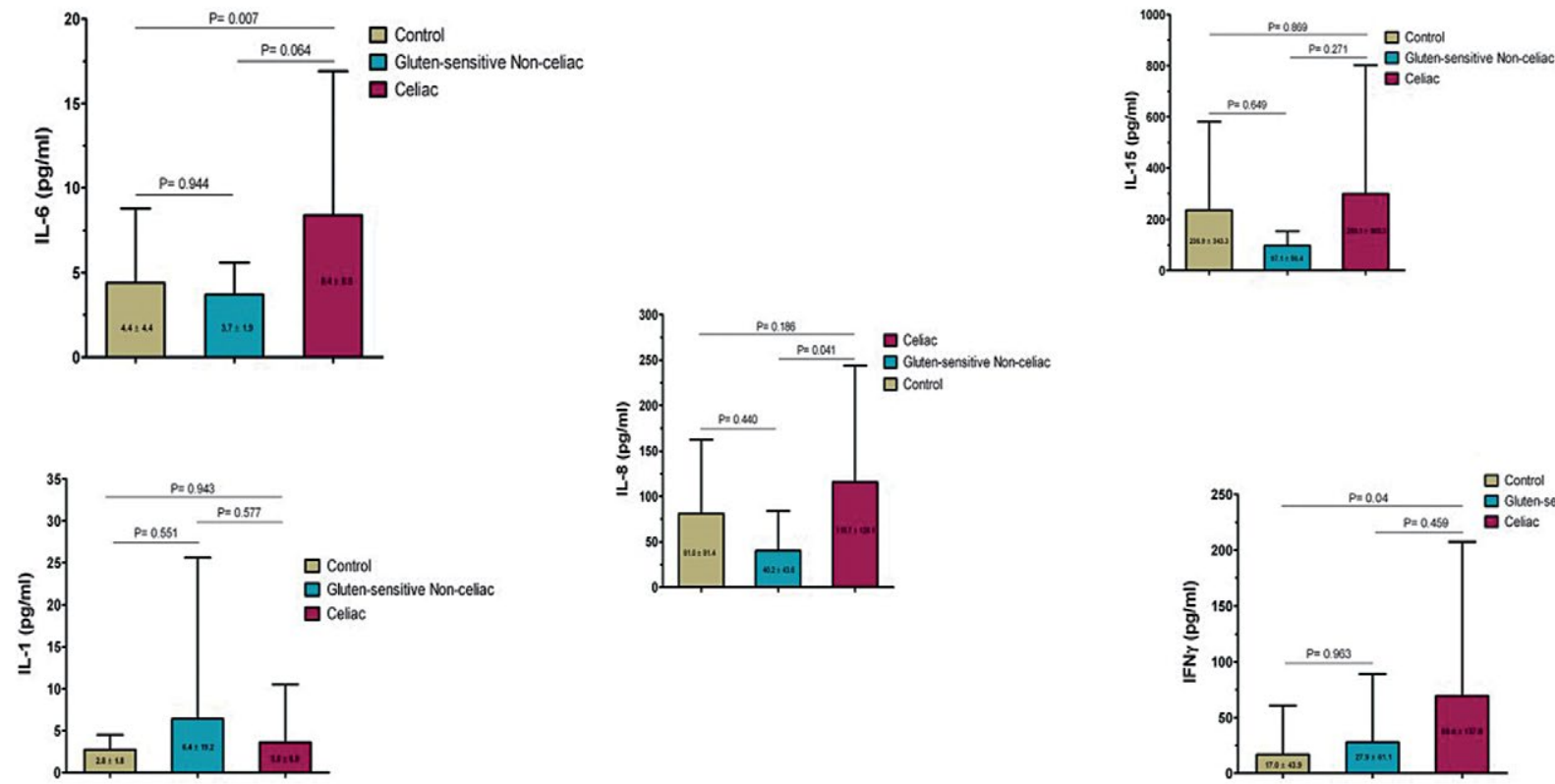

Fig. 1. Serum levels of inflammatory cytokines (IL-1, IL-15, IFN- $\gamma$ and IL-8) and pro-inflammatory cytokine (IL-6) in CD, NCGS and control groups (Data are presented as mean values of group $\pm \mathrm{SD}$. Statistically significant differences were tested with one-way ANOVA and the Tukey's Multiple Comparison Test; $\left.{ }^{*} \mathrm{p}<0.05\right)$. 
showed that the mean serum levels of IFN- $\gamma(p=0.04)$ and IL-6 ( $p=0.007)$ were significantly different between the CD and control groups. It is noteworthy that the mean serum level of IL-8 was significantly higher in CD patients in comparison to the NCGS group ( $p=0.041$; Fig. 1 ).

Despite the higher mean serum levels of IL-1 in NCGS patients, as compared with patients with CD and healthy subjects, this difference was not statistically significant $(\mathrm{p}=0.943)$. Interestingly, although the higher mean serum levels of IL-15 in patients in the CD group (69.4 \pm 137.9$)$ as compared with patients in the NCGS $(27.9 \pm 61.1)$ and control $(17.0 \pm$ 43.9) groups, these differences were not significantly different between the studied populations $(\mathrm{p}=0.869)$.

Regarding the correlation between symptoms and investigated cytokine levels, statistically significant correlations were observed in the serum levels of IFN $-\gamma$ with abortion $(\mathrm{p}=0.01)$ and serum levels of IL-1 with weight loss ( $\mathrm{p}=$ $0.043)$ and infertility $(\mathrm{p}=0.0001)$ in CD patients (Table II). Moreover, there was a statistically significant correlation between the serum levels of IFN- $\gamma$ with abortion $(\mathrm{p}=0.01)$ and infertility $(\mathrm{p}=0.01)$ in NCGS patients. No statistically significant correlation was reported between the mode of any other clinical presentations and other studied cytokines in CD/ NCGS patients. On the other hand, there was no significant relationship between the severity of mucosal damage and the serum level of studied cytokines in CD patients $(\mathrm{p}=0.41)$.

\section{DISCUSSION}

Our findings indicate that the inflammatory responses in CD may be characterized by the elevated levels of IFN- $\gamma$ and IL-6, which can be considered as Th1- and Th2-derived cytokines, respectively [24]. Dienz and Rincon [24] showed that IL-6 can modulate the Th1/Th2 balance toward Th2. The important aspects of the proliferation and variation of IL- 6 belong to the differentiation capacity of $\mathrm{B}$ lymphocytes to produce immunoglobulin and activate T cells [24]. Although elevations in certain cytokines are comparable between local gut mucosa and systematic serum levels, some disparities can be observed by non-intestinal sources. It has been well demonstrated that

Table II. The correlations between studied cytokines and clinical symptoms in CD and NCGS patients.

\begin{tabular}{|c|c|c|c|c|c|c|}
\hline \multirow[b]{2}{*}{ Clinical parameters } & \multicolumn{6}{|c|}{$\mathrm{p}$ values ${ }^{*}$} \\
\hline & Groups & $\begin{array}{c}\text { IL-15 } \\
\text { (ng/mL) }\end{array}$ & $\begin{array}{c}\text { IL-8 } \\
\text { (ng/mL) }\end{array}$ & $\begin{array}{c}\text { IL-6 } \\
(\mathrm{ng} / \mathrm{mL})\end{array}$ & $\begin{array}{c}\mathrm{IL}-1 \\
(\mathrm{ng} / \mathrm{mL})\end{array}$ & $\begin{array}{c}\text { IFN- } \gamma \\
(\mathrm{ng} / \mathrm{mL})\end{array}$ \\
\hline \multirow{2}{*}{ Diarrhea } & $\mathrm{CD}$ & 0.36 & 0.636 & 0.334 & 0.572 & 0.492 \\
\hline & NCGS & 0.46 & 0.371 & 0.715 & 0.207 & 0.382 \\
\hline \multirow[t]{2}{*}{ Bloating } & $\mathrm{CD}$ & 0.432 & 0.732 & 0.847 & 0.875 & 0.987 \\
\hline & NCGS & 0.482 & 0.604 & 0.415 & 0.241 & 0.521 \\
\hline \multirow[t]{2}{*}{ Gas passing } & $\mathrm{CD}$ & 0.441 & 0.589 & 0.611 & 0.771 & 0.665 \\
\hline & NCGS & 0.482 & 0.604 & 0.187 & 0.501 & 0.421 \\
\hline \multirow[t]{2}{*}{ Crumping } & $\mathrm{CD}$ & 0.418 & 0.545 & 0.603 & 0.4 & 0.13 \\
\hline & NCGS & 0.446 & 0.286 & 0.139 & 0.112 & 0.306 \\
\hline \multirow[t]{2}{*}{ Weight loss } & $\mathrm{CD}$ & 0.349 & 0.797 & 0.072 & 0.043 & 0.392 \\
\hline & NCGS & 0.449 & 0.359 & 0.254 & 0.787 & 0.426 \\
\hline \multirow[t]{2}{*}{ Nausea and vomiting } & $\mathrm{CD}$ & 0.455 & 0.456 & 0.405 & 0.623 & 0.234 \\
\hline & NCGS & 0.301 & 0.359 & 0.243 & 0.664 & 0.323 \\
\hline \multirow[t]{2}{*}{ Anemia } & $\mathrm{CD}$ & 0.448 & 0.302 & 0.398 & 0.525 & 0.699 \\
\hline & NCGS & 0.46 & 0.132 & 0.686 & 0.821 & 0.504 \\
\hline \multirow[t]{2}{*}{ Bone disease } & $\mathrm{CD}$ & 0.432 & 0.374 & 0.46 & 0.454 & 0.595 \\
\hline & NCGS & 0.46 & 0.398 & 0.584 & 0.159 & 0.204 \\
\hline \multirow[t]{2}{*}{ Neurological symptoms } & $\mathrm{CD}$ & 0.374 & 0.623 & 0.468 & 0.322 & 0.514 \\
\hline & NCGS & 0.449 & 0.448 & 0.434 & 0.464 & 0.425 \\
\hline \multirow[t]{2}{*}{ Menstrual disorder } & $\mathrm{CD}$ & 0.465 & 0.105 & 0.183 & 0.556 & 0.523 \\
\hline & NCGS & 0482 & 0.345 & 0.392 & 0.832 & 0.521 \\
\hline \multirow[t]{2}{*}{ Infertility } & $\mathrm{CD}$ & 0.25 & 0.657 & 0.595 & 0.000 & 0.145 \\
\hline & NCGS & 0.301 & 0.132 & 0.545 & 0.848 & 0.016 \\
\hline \multirow[t]{2}{*}{ Abortion } & $\mathrm{CD}$ & 0.25 & 0.144 & 0.733 & 0.7 & 0.01 \\
\hline & NCGS & 0.301 & 0.646 & 0.132 & 0.703 & 0.01 \\
\hline \multirow[t]{2}{*}{ Aphtus } & $\mathrm{CD}$ & 0.349 & 0.325 & 0.35 & 0.521 & 0.831 \\
\hline & NCGS & 0.489 & 0.256 & 0.884 & 0.201 & 0.521 \\
\hline \multirow[t]{2}{*}{ Skin disease } & $\mathrm{CD}$ & 0.746 & 0.451 & 0.972 & 0.15 & 0.608 \\
\hline & NCGS & 0.332 & 0.132 & 0.241 & 0.703 & 0.256 \\
\hline
\end{tabular}


gliadin induces the production of proinflammatory cytokines that are involved in modulating the Th1/Th2 balance, which in turn enhances cell-mediated immunity [25]. On the other hand, Th2-derived cytokines essentially affect the humoral immune response and repress inflammatory responses [26]. The elevated serum levels of IFN- $\gamma$, IL-6, and IL- 8 , which have been shown to be high in the gut mucosa, suggest that CD induces secretion and systemic activation of these cytokines. As an important mediator of Th1 immune response, IFN- $\gamma$ was constantly elevated in CD patients, and numerous studies have also suggested the coincidence of IFN- $\gamma$ elevation in lamina propria with histological changes in the small intestine [27]. Elevated levels of IFN- $\gamma$ in CD patients, even after 6 months of strict GFD, suggest that the remnant of intestinal epithelial abnormality keeps the indicated cytokine at a high level, whereas in the NCGS group, epithelial changes are more subtle; the low levels of serum IFN- $\gamma$ in this group can be explained according to these phenomena. Many studies have shown that the investigation of villous atrophy in NCGS subjects was indicative of mucosal architectural abnormalities [28]. Abed et al. [29] evaluated the serum level of IFN- $\gamma$ in 50 Iraqi patients with CD and 50 control patients and reported that the level of IFN- $\gamma$ is significantly higher in the patients with CD than in the control patients, and the mean level of IFN- $\gamma$ significantly decreased after 6 months of GFD. Manavalan et al. evaluated the serum levels of cytokines in patients with active $C D$, seropositive and seronegative patients on a GFD, refractory CD, and healthy subjects [18]. Their results showed that patients with active $\mathrm{CD}$ and seropositive patients on a GFD had significantly higher levels of IFN- $\gamma$ compared with normal controls and other groups. In accordance with our findings, Di Sabatino et al. [28] recently showed that the serum and mRNA levels of IFN- $\gamma$ were significantly higher in patients with untreated $\mathrm{CD}$ than in patients with treated $\mathrm{CD}$, patients with self-reported NCGS, and controls. It was also confirmed that the IFN- $\gamma$ levels in the duodenal mucosal tissue of the CD group patients were much higher than those of the patients in the control and NCGS groups [30]. Losurdo et al. [30] showed that of 89 patients with microscopic enteritis (ME) of different etiology, CD patients had the highest IFN- $\gamma$ levels than other studied groups.

Similar to IFN- $\gamma$, IL-15 is also a proinflammatory cytokine and many studies were suggestive of its role in celiac pathogenesis. Previous studies have also shown the pivotal role of this cytokine in the pathogenesis of epithelial lesions [31]. An increased level of IL-15 was reported in the serum of patients with autoimmune disorders, such as IBD, systemic lupus erythematosus, type 1 diabetes, and rheumatoid arthritis [31-34]. Remarkably, the expression of IL-15 diverges between different types of CD such as active, potential, and patients undergoing GFD. A previous study confirmed that patients with active CD have increased levels of IL-15 in the small intestinal mucosa [28]. On the other hand, it was proposed [35] that the dysregulation of IL-15 expression in the epithelium of CD patients on a GFD was inadequate to induce villous atrophy. Di Sabatino et al. [28] also reported a significantly increased level of IL-15 in patients with untreated CD compared with those in other studied groups. Furthermore, Abed et al. [29] confirmed the significantly increased level of IL-15 in patients with untreated CD compared with those in the control group.
Contrary to the elevation of IL-15 in damaged mucosa, it seems that elevation in serum IL-15 is barely visible when comparing CD subjects with healthy controls. Moreover, no IL-15 elevation was observed in the NCGS group. Therefore, these results are indicative of the local elevation of IL-15 and suggestive of its involvement in the pathogenesis of glutentriggered complications.

Likewise, significant differences were not observed in the serum levels of IL- $1 \alpha$ and IL- $1 \beta$ in all groups. It is possible that the variation observed in the serum levels of these cytokines might be due to the differences in their origins, structures, enzymes involved in the conversion of these two homologous cytokines into active peptides, and their circulating antagonist receptors [10]. Similar to our finding, Di Sabatino et al. [28] did not find a significant difference in the levels of IL- $1 \beta$ between the study populations. In contrast, Manavalan et al. [20] reported a significantly higher level of IL-1 $\beta$ in the NCGS group than in the control group.

IL-8, as a chemokine expressed in tissues with neutrophilic infiltration, engaging in inflammatory responses mediated by neutrophils [36], has shown a significant increase in the CD group, even years after the exclusion of gluten from the diet, as compared with the healthy control and NCGS groups. Contrary to the results of IL- 8 in the CD group in this study, results of previous studies were indicative of a significant decrease in the serum level of IL- 8 of dermatitis herpetiformis subjects after one year of GFD [37]. Similar to our study, a significantly higher amount of IL- 8 was reported in patients with untreated CD compared with those in the NCGS, treated CD, and control groups [28]. The underlying cause of this phenomenon is yet to be discovered, but we believe that it may have resulted from the prolonged activation of proinflammatory responses in patients with CD in the small intestine.

As a pleiotropic cytokine, IL- 6 can be produced by heterogeneous cell populations, including endothelial cells, fibroblasts, monocytes, B cells, T cells, keratinocytes, and limited number of tumors [38]. IL-6 participates in the immune regulation, inflammation, hematopoiesis, and oncogenesis, thus playing a pivotal role in the pathogenesis of inflammationrelated disorders [39]. Interestingly, results of the previous studies are indicative of a significant decrease in the serum levels of IL- 6 after 6 months to 1 year of GFD [10, 40], contrary to the significant increase in the serum levels of IL- 6 in patients in the CD group of our study despite 1 year of GFD. Di Sabatino et al. [28] and Manavalan et al. [20] confirmed that the level of IL-6 is higher in CD patients than in NCGS and control group patients, respectively. The serum levels of IL- 6 in the patients in the NCGS group are lower because of the different pathogenesis of the disease [41]; in the NCGS group, IL-6 plays an important role only in the case of non-GI symptoms, when compared with the CD group, in which the serum level of IL- 6 remains elevated because of the systemic involvement of inflammation.

Although the systemic involvement and inflammation cannot be measured by assays at this time, our results suggest different roles of certain cytokines, especially those of IL- 6 and IL-8 in sustaining humoral responses. Increased levels of serum cytokines in patients with severe mucosal damage suggest a link between mucosal alteration and systemic immune activation in CD patients, more extensively than in NCGS patients. 
It is more likely that the systemic immune activation might be the cause of extra-GI manifestations of CD. A recent study proposed that the imbalance of Th1/Th2 cytokines is an important determinant in pregnancy maintenance as well as abortion [42]. Ma et al. [42] reported that IL-1 $\beta$ and IL-6 gene polymorphisms may be proposed as an important risk factor and protect factor, respectively, against the risk of recurrent abortion. This is in concordance with the results of this study, in which statistically significant correlations have been observed between the serum levels of IFN- $\gamma$ and abortion in CD and NCGS groups (each $p=0.01$ respectively) and serum levels of IFN- $\gamma$ and infertility in NCGS patients $(\mathrm{p}=0.01)$. A significant correlation was also observed between the serum levels of IL-1 and weight loss $(\mathrm{p}=0.043)$ and infertility $(\mathrm{p}=0.0001)$ in CD patients. It is worth mentioning that the presence of IFN- $\gamma$ and IL-1 receptors could lead to false low levels, and in turn, the simultaneous presence of other inflammatory processes may elevate levels of certain cytokines. However, the consistent elevation of certain cytokines, such as IFN- $\gamma$ and IL-1, in CD patients may be indicative of the fact that these considerations do not affect our results substantially. The main limitation of this study was the difference in the number of people in the groups.

Our study showed that the mean serum levels of IFN- $\gamma$ and IL-6 were significantly different between the CD and control groups. Comparing the profile of serum cytokines in CD patients, only IL-8 was significantly different in the NCGS group. Interestingly, the mentioned cytokines were significantly associated with different GI and extra-GI symptoms when we compared the CD and NCGS groups. Further studies should concentrate on determining immunological biomarkers to illustrate what is possibly dissimilar between the CD and NCGS groups and between the NCGS and healthy control groups. Furthermore, to find an effective biomarker for NCGS diagnosis [43], more studies investigating this aspect are essential.

\section{CONCLUSION}

The elevation in serum levels of IL- 8 in CD subjects can potentially be used for differentiating NCGS from CD, and the evaluation of IL-1 is proposed as a distinguishing marker between the NCGS and control groups. A practical, comprehensive pattern of the cytokines involved in CD and NCGS not only provides an insight into the pathogenesis, symptoms, and clinical manifestations of CD, but it also leads to the development of better tools to monitor these disorders.

Conflicts of interest: No conflict to declare.

Authors' contributions: M.R.N. and E.M.H. conceived and designed the experiments; F.H. and E.A. performed the experiments; M.A.P. and A.M.A. analyzed the data; K.R., M.R.Z. contributed with reagents/ materials/analysis tools; F.H. and M.R.N. wrote the manuscript. All the authors approved the final version.

Acknowledgments: This study was supported by the Gastroenterology and Liver Disease Research Center, Shahid Beheshti University of Medical Sciences, Tehran, Iran. We thank Mrs. Shima Rasouli and other colleagues in celiac disease research department, for providing insight and expertise that greatly assisted this research.

\section{REFERENCES}

1. Rostami Nejad M, Rostami K, Pourhoseingholi MA, et al. Atypical presentation is dominant and typical for coeliac disease. J Gastrointestin Liver Dis 2009;8:285-291.

2. Rostami-Nejad M, Villanacci V, Hogg-Kollars, et al. Endoscopic and histological pitfalls in the diagnosis of celiac disease: A multicentre study assessing the current practice. Rev Esp Enferm Dig 2013;105:326-333.

3. Tursi A, Brandimarte G, Giorgetti G.M. Prevalence of antitissue transglutaminase antibodies in different degrees of intestinal damage in celiac disease. J Clin Gastroenterol 2003;36:219-21.

4. Hisamatsu T, Erben U, Kühl AA. The Role of T-Cell Subsets in Chronic Inflammation in Celiac Disease and Inflammatory Bowel Disease Patients: More Common Mechanisms or More Differences? Inflamm Intest Dis 2016;1:52-62. doi:10.1159/000445133

5. Mazzarella G. Effector and suppressor T cells in celiac disease. World J Gastroenterol 2015;21:7349-7356. doi:10.3748/wjg.v21.i24.7349

6. Hüe S, Mention JJ, Monteiro RC, et al. A direct role for NKG2D/ MICA interaction in villous atrophy during celiac disease. Immunity 2004;21:367-377. doi:10.1016/j.immuni.2004.06.018

7. Mashayekhi K, Rostami-Nejad M, Azimzadeh P, et al. Setup of SYBR green real-time PCR method to detect the HLA-DQ alleles in patients with celiac disease. Koomesh 2015;16:527-535.

8. Schuppan D, Junker Y, Barisani D. Celiac disease: from pathogenesis to novel therapies. Gastroenterology 2009;137:1912-1933. doi:10.1053/j. gastro.2009.09.008

9. Meresse B, Chen Z, Ciszewski C, et al. Coordinated induction by IL15 of a TCR-independent NKG2D signaling pathway converts CTL into lymphokine-activated killer cells in celiac disease. Immunity 2004; 21:357-366. doi:10.1016/j.immuni.2004.06.020

10. Fornari MC, Pedreira S, Niveloni $S$, et al. Pre-and post-treatment serum levels of cytokines IL- $1 \beta$, IL-6, and IL-1 receptor antagonist in celiac disease. Are they related to the associated osteopenia? Am J Gastroenterol 1998;93:413-418. doi:10.1111/j.1572-0241.1998.00413.x

11. Daum S, Bauer U, Foss HD, et al. Increased expression of mRNA for matrix metalloproteinases-1 and-3 and tissue inhibitor of metalloproteinases- 1 in intestinal biopsy specimens from patients with coeliac disease. Gut 1999;44:17-25.

12. Catassi C, Elli L, Bonaz B, et al. Diagnosis of non-celiac gluten sensitivity (NCGS): the Salerno experts' criteria. Nutrients 2015;7:4966-4977. doi:10.3390/nu7064966

13. SaponeA, Bai JC, Ciacci C, et al. Spectrum of gluten-related disorders: Consensus on new nomenclature and classification. BMC Med 2012;10:13. doi:10.1186/1741-7015-10-13

14. Rostami K, Hogg-Kollars S. A Patient's Journey. Non-coeliac gluten sensitivity. BMJ 2012;345:e7982. doi:10.1136/bmj.e7982

15. Shahbazkhani B, Sadeghi A, Malekzadeh R, et al. Non-celiac gluten sensitivity has narrowed the spectrum of irritable bowel syndrome: a double-blind randomized placebo-controlled trial. Nutrients 2015;7:4542-4554. doi:10.3390/nu7064542

16. Biesiekierski JR, Peters SL, Newnham ED, Rosella O, Muir JG, Gibson PR. No effects of gluten in patients with self-reported non-celiac gluten sensitivity after dietary reduction of fermentable, poorly absorbed, short-chain carbohydrates. Gastroenterology 2013;145: 320-328.e1-e3. doi:10.1053/j.gastro.2013.04.051 
17. Ludvigsson JF, Leffler DA, Bai JC, et al. The Oslo definitions for coeliac disease and related terms. Gut 2013;62:43-52. doi:10.1136/ gutjnl-2011-301346

18. Peña AS. Immunogenetics of non celiac gluten sensitivity. Gastroenterol Hepatol Bed Bench 2014;7:1-5.

19. Iftikhar R, Jamal S, Zafar A, Saadia A. Histopathological and Immunohistochemical Analysis of Small Intestinal Biopsies in Adults Suspected of Celiac Disease. J Coll Physicians Surg Pak 2016;26:827-830.

20. Manavalan JS, Hernandez L, Shah JG, et al. Serum cytokine elevations in celiac disease:association with disease presentation. Hum Immunol 2010;71:50-57. doi:10.1016/j.humimm.2009.09.351

21. Salvati VM, Mazzarella G, Gianfrani C, et al. Recombinant human interleukin 10 suppresses gliadin dependent $\mathrm{T}$ cell activation in ex vivo cultured coeliac intestinal mucosa. Gut 2005;54:46-53. doi:10.1136/ gut.2003.023150

22. Cinova J, Palová-Jelínková L, Smythies L.E, et al. Gliadin peptides activate blood monocytes from patients with celiac disease. J Clin Immunol 2007;27:201-209. doi:10.1007/s10875-006-9061-Z

23. Marsh MN, Johnson MW, Rostami K. Mucosal histopathology in celiac disease: a rebuttal of Oberhuber's sub-division of Marsh III. Gastroenterol Hepatol Bed Bench 2015;8:99-109.

24. Dienz O, Rincon M. The effects of IL- 6 on CD4 T cell responses. Clin Immunol 2009;130:27-33. doi:10.1016/j.clim.2008.08.018

25. Ben-Horin S, Green PH, Bank I, Chess L, Goldstein I. Characterizing the circulating, gliadin-specific CD4+ memory $\mathrm{T}$ cells in patients with celiac disease: linkage between memory function, gut homing and Th1 polarization. J Leukoc Biol 2006;79:676-685. doi:10.1189/jlb.0705414

26. McSorley HJ, Gaze S, Daveson J, et al. Suppression of inflammatory immune responses in celiac disease by experimental hookworm infection. PLoS One 2011;6:e24092. doi:10.1371/journal.pone.0024092

27. Ferber IA, Brocke S, Taylor-Edwards C, et al. Mice with a disrupted IFN-gamma gene are susceptible to the induction of experimental autoimmune encephalomyelitis (EAE). J Immunol 1996;156:5-7.

28. Di Sabatino A, Giuffrida P, Fornasa G, et al. Innate and adaptive immunity in self-reported nonceliac gluten sensitivity versus celiac disease. Dig Liver Dis 2016;48:745-752. doi:10.1016/j.dld.2016.03.024

29. Abed RM, Yaaqoob LA, Alwan IA, Samir HF, Jasim RA. Evaluation of some Serum cytokine and adipokines profile in Iraqi celiac patients before and after treatment. IOSR J Pharm Biol Sci 2017;12:36-41.

30. Losurdo G, Giorgio F, Piscitelli D, et al. May the assessment of baseline mucosal molecular pattern predict the development of gluten related disorders among microscopic enteritis? World J Gastroenterol 2016;22:8017-8025. doi:10.3748/wjg.v22.i35.8017

31. Di Sabatino A, Ciccocioppo R, Cupelli F, et al. Epithelium derived interleukin 15 regulates intraepithelial lymphocyte Th1 cytokine production, cytotoxicity, and survival in coeliac disease. Gut 2006;55:469-477. doi:10.1136/gut.2005.068684

32. Losy J, Niezgoda A, Zaremba J. IL-15 is elevated in sera of patients with relapsing-remitting multiple sclerosis. Folia Neuropathol 2002;40:151153.

33. Aringer M, Stummvoll GH, Steiner G, et al. Serum interleukin-15 is elevated in systemic lupus erythematosus. Rheumatology (Oxford) 2001;40:876-881. doi:10.1093/rheumatology/40.8.876

35. Kuczynski S, Winiarska H, Abramczyk M, Szczawinska K, WieruszWysocka B, Dworacka M. IL-15 is elevated in serum patients with type 1 diabetes mellitus. Diabetes Res Clin Pract 2005;69:231-236. doi:10.1016/j.diabres.2005.02.007

36. Abadie V, Jabri B. IL-15: a central regulator of celiac disease immunopathology. Immunol Rev 2014;260:221-234. doi:10.1111/ imr.12191

37. Li A, Dubey S, Varney ML, Dave BJ, Singh RK. IL-8 directly enhanced endothelial cell survival, proliferation, and matrix metalloproteinases production and regulated angiogenesis. J Immunol 2003;170:3369-3376. doi:10.4049/jimmunol.170.6.3369

38. Hall RP 3rd, Takeuchi F, Benbenisty KM, Streilein RD. Cutaneous endothelial cell activation in normal skin of patients with dermatitis herpetiformis associated with increased serum levels of IL-8, sE-selectin, and TNF-a. J Invest Dermatol 2006;126:1331-1337. doi:10.1038/ sj.jid. 5700277

39. Grivennikov S, Karin E, Terzic J, et al. IL-6 and Stat3 are required for survival of intestinal epithelial cells and development of colitisassociated cancer. Cancer Cell 2009;15:103-113. doi:10.1016/j. ccr.2009.01.001

40. Lesina M, Kurkowski MU, Ludes K, et al. Stat3/Socs3 activation by IL-6 transsignaling promotes progression of pancreatic intraepithelial neoplasia and development of pancreatic cancer. Cancer Cell 2011;19:456-469. doi:10.1016/j.ccr.2011.03.009

41. Sapone A, Lammers KM, Casolaro V, et al. Divergence of gut permeability and mucosal immune gene expression in two glutenassociated conditions: celiac disease and gluten sensitivity. BMC Med 2011;9:23. doi:10.1186/1741-7015-9-23

42. Catassi C, Bai JC, Bonaz B, et al. Non-Celiac Gluten sensitivity: the new frontier of gluten related disorders. Nutrients 2013;5:3839-3853. doi:10.3390/nu5103839

43. Ma J, Zhang X, He G, Yang C. Association between TNF, IL1B, IL6, IL10 and IFNG polymorphisms and recurrent miscarriage: a case control study. Reprod Biol Endocrinol 2017;15:83. doi:10.1186/s12958-017-0300-3

44. Ierardi E, Losurdo G, Piscitelli D, et al. Biological markers for nonceliac gluten sensitivity: a question awaiting for a convincing answer. Gastroenterol Hepatol Bed Bench 2018;11:203-208. 